\title{
TU/e EmonONEN

\section{Solidification in closed systems: cluster size distribution and its driving force}

Citation for published version (APA):

Hütter, M. (2003). Solidification in closed systems: cluster size distribution and its driving force. Multiscale Modeling \& Simulation, 1(3), 371-390. https://doi.org/10.1137/S1540345902416107

DOI:

10.1137/S1540345902416107

Document status and date:

Published: 01/01/2003

\section{Document Version:}

Publisher's PDF, also known as Version of Record (includes final page, issue and volume numbers)

\section{Please check the document version of this publication:}

- A submitted manuscript is the version of the article upon submission and before peer-review. There can be important differences between the submitted version and the official published version of record. People interested in the research are advised to contact the author for the final version of the publication, or visit the $\mathrm{DOI}$ to the publisher's website.

- The final author version and the galley proof are versions of the publication after peer review.

- The final published version features the final layout of the paper including the volume, issue and page numbers.

Link to publication

\section{General rights}

Copyright and moral rights for the publications made accessible in the public portal are retained by the authors and/or other copyright owners and it is a condition of accessing publications that users recognise and abide by the legal requirements associated with these rights.

- Users may download and print one copy of any publication from the public portal for the purpose of private study or research.

- You may not further distribute the material or use it for any profit-making activity or commercial gain

- You may freely distribute the URL identifying the publication in the public portal.

If the publication is distributed under the terms of Article 25fa of the Dutch Copyright Act, indicated by the "Taverne" license above, please follow below link for the End User Agreement:

www.tue.nl/taverne

Take down policy

If you believe that this document breaches copyright please contact us at:

openaccess@tue.nl

providing details and we will investigate your claim. 


\title{
SOLIDIFICATION IN CLOSED SYSTEMS: CLUSTER SIZE DISTRIBUTION AND ITS DRIVING FORCE*
}

\author{
MARKUS HÜTTER ${ }^{\dagger}$
}

\begin{abstract}
A multiphase model for liquid-solid and solid-liquid phase transitions is developed using the framework of the general equation for the nonequilibrium reversible-irreversible coupling (GENERIC). The morphology is described by the cluster size distribution function, including both unstable embryos as well as stable crystallites, for which a diffusion equation is proposed. The driving forces therein are identified in terms of the thermodynamic states of the two competing phases and of the surface tension. The connection to a previously developed model, which considered only the zeroth to third moments of the distribution function, is established. Whereas the growth rates of the crystallites are similar, the striking difference is the expression for the nucleation rate. The latter is furnished by the diffusion of clusters over a potential barrier in the model developed here, whereas such effects are absent in the previous model.
\end{abstract}

Key words. cluster size distribution, solidification, phase transition, multiphase model

AMS subject classifications. 80A20, 82C05, 82C26, 82C31, 82C35

DOI. $10.1137 / \mathrm{S} 1540345902416107$

\section{Introduction: Problem statement.}

1.1. Kolmogorov-Johnson-Mehl-Avrami theory and beyond. The process of crystallization of a melt can be considered from different perspectives and on various levels of description. However, the theory to determine the overall crystallization as developed by Kolmogorov [1], Johnson and Mehl [2], and Avrami [3, 4, 5] is ubiquitous. It relates the volume fraction of the crystalline phase $\phi(t)$ at time $t$ to the nucleation rate per unit volume $\alpha(t)\left[\mathrm{m}^{-3} \mathrm{~s}^{-1}\right]$ through

$$
\phi(t)=1-\exp \left(\int_{-\infty}^{t} \alpha\left(t^{\prime}\right) v\left(t^{\prime}, t\right) d t^{\prime}\right)
$$

where $v\left(t^{\prime}, t\right)$ denotes the volume at time $t$ of a single crystallite which began to grow from a nucleus at time $t^{\prime}$. The term in the exponent of (1.1) is known as the extended volume fraction, which can grow beyond any limits, in contrast to the physical volume fraction $\phi$ with $0 \leq \phi \leq 1$.

The relation between the extended and the physical volume fraction as given in (1.1) has its mathematical background in the Boolean grain model often studied in integral geometry (see, e.g., [6]). In integral geometry in general, it is found that the natural number of integral measures to characterize a two-dimensional manifold in three-dimensional space is four, the measures being the Minkowski functionals, also known as quermass integrals, or curvature integrals. The measures consist of the volume, the surface, a characteristic length scale, and the Euler characteristic function, characterizing the morphology and topology, respectively. The Boolean grain model on its turn then allows us to express the Minkowski functionals for the union of randomly placed and oriented and arbitrarily overlapping convex grains,

\footnotetext{
* Received by the editors October 15, 2002; accepted for publication (in revised form) May 14, 2003; published electronically July 17, 2003.

http://www.siam.org/journals/mms/1-3/41610.html

${ }^{\dagger}$ Institute of Polymers, ETH Zentrum, ML-H18, Sonneggstr. 3, CH-8092 Zürich, Switzerland. Current address: Department of Chemical Engineering, Massachusetts Institute of Technology, Room 66-258, 77 Massachusetts Avenue, Cambridge, MA 02139-4307 (mhuetter@mit.edu).
} 
e.g., impinging crystallites, in terms of the Minkowski functionals of the single grains. Hence, for given growth behavior of the single grains, the growth of the Minkowski functionals of the overall structure results accordingly. ${ }^{1}$

The advantage of considering the Boolean grain model for crystallization clearly is in the reduction of studying only the growth of convex bodies of much simpler shape than that of the overall structure. This has exactly been used by Schneider, Köppel, and Berger [7] to transform the integral equation (1.1) into a set of four coupled differential equations for the specific situation of spherical grains. Rather than writing equations for the Minkowski functionals of the union of grains, they computed the dynamic equations for the averages of single-grain Minkowski measures (with slightly different prefactors), namely, for

$$
\Psi_{\mu}(t):=n(t) \frac{8 \pi}{(3-\mu) !}\left\langle R^{(3-\mu)}\right\rangle_{p(t)} \quad(\mu=0,1,2,3),
$$

where $n(t)$ is the number of spherical crystallites per unit volume, and $\langle\ldots\rangle_{p(t)}$ denotes the average with respect to the size distribution $p(R, t)$ (normalized to unity) at instant $t$. One finds that the $\Psi_{\mu}$ are the average grain volume $(\mu=0)$, the average grain surface $(\mu=1), 8 \pi$ times the average grain radius $(\mu=2)$, and $8 \pi(\mu=3)$, all multiplied by the number density $n(t)$. If the radial growth rate of the spherulites is given by $G(t)\left[\mathrm{m} \mathrm{s}^{-1}\right]$ and the nucleation rate per unit volume by $\alpha(t)$, the so-called Schneider rate equations for the quantities defined in $(1.2)$ then read $[7,8,9]$

$$
\begin{aligned}
& \partial_{t} \Psi_{\mu}(t)=G(t) \Psi_{\mu+1}(t) \quad(\mu=0,1,2), \\
& \partial_{t} \Psi_{3}(t)=8 \pi \alpha(t) .
\end{aligned}
$$

From an integral geometric point of view, on the one hand, it is satisfactory to see that the number of dynamic variables is the desired four. On the other hand, however, for a physical description of crystallization, in particular the physical volume fraction $\phi$ and the physical amount of interface per unit volume $\psi$ are more desirable quantities than $\Psi_{0}$ and $\Psi_{1}$, respectively. Using the Boolean grain model, the appropriate transformations are given by

$$
\begin{aligned}
& \phi=1-e^{-\Psi_{0}}, \\
& \psi=e^{-\Psi_{0}} \Psi_{1} .
\end{aligned}
$$

The "physical" analogons to $\Psi_{2}$ and $\Psi_{3}$, namely the average mean curvature and the connectivity (see, e.g., [6]), are not introduced here, as they will not be involved in the following discussion. Thus, the dynamic equations for morphology changes due to phase transformation in terms of the variables

$$
\mathbf{\Phi} \equiv\left(\phi, \psi, \Psi_{2}, \Psi_{3}\right),
$$

which are of primary interest in the further study, follow as

$$
\begin{aligned}
\partial_{t} \phi & =G(t) \psi, \\
\partial_{t} \psi & =G(t) \mathcal{L}\left(\phi, \psi, \Psi_{2}\right), \\
\partial_{t} \Psi_{2} & =G(t) \Psi_{3}, \\
\partial_{t} \Psi_{3} & =8 \pi \alpha(t),
\end{aligned}
$$

\footnotetext{
${ }^{1}$ In contrast to crystallization, the assumption of overlapping/impinging grains is less appropriate for condensation phenomena. There, two growing droplets coalesce upon contact rather than overlap/impinge, which results in a bigger droplet of again spherical shape. Such coalescence events are not captured by the model discussed here. To do so would mean to replace the Boolean grain model only, leaving the rest of the treatment unchanged.
} 
with the length scale

$$
\mathcal{L}\left(\phi, \psi, \Psi_{2}\right):=\left(\Psi_{2}-\Psi_{1}^{2}\right) e^{-\Psi_{0}}=(1-\phi)\left(\Psi_{2}-\frac{\psi^{2}}{(1-\phi)^{2}}\right),
$$

where (1.3a) and the relations (1.4a), (1.4b) have been employed. For later convenience, the set of equations (1.6a)-(1.6d) shall be summarized as

$$
\left.\partial_{t} \Phi_{\mu}\right|_{\mathrm{pc}}=\Upsilon_{\mu}(\mathbf{x}) \quad(\mu=0,1,2,3) .
$$

The subscript "pc" denotes the rate of change in the variables (1.5) due to phase change, and the argument $\mathbf{x}$ of the functions $\Upsilon_{\mu}$ indicates arbitrary dependence on any variables included in the description.

The dynamic equations (1.6a)-(1.6d) or (1.8), respectively, for the morphological descriptors become physical only when specifying the radial growth rate $G$ and the nucleation rate $\alpha$. For practical purposes, these can be taken from experimental data under standard conditions. However, gaining a fundamental understanding of their origin amounts to identifying the driving forces for phase change. In the present manuscript, a relation between $G$ and $\alpha$, on the one hand, to the difference in thermodynamic states between the two competing phases, on the other hand, is established. This shall be achieved by using a multiphase model to capture the evolution of the thermodynamic states of the phases and combining it with the rate equations (1.6a)-(1.6d) by using a nonequilibrium thermodynamics formalism. The manuscript is organized as follows. In the remainder of the introduction, the multiphase model is described, as well as the nonequilibrium thermodynamics formalism GENERIC. Then the specific problem of a previously developed model for phase change is discussed, which results in formulating the goal of the present study. The introduction is intentionally rather detailed, as this, first, allows for a transparent motivation of the goal and, second, considerably simplifies the development of the new model in section 2. Consequences of the model are studied in section 3 , followed by a discussion and conclusions in section 4 .

1.2. Multiphase model. In view of establishing a connection between the radial growth rate $G$ and the nucleation rate $\alpha$ and the thermodynamic state of the competing phases, the appropriate variables need to be included in the full description. To that end, it is essential to note that the spatial resolution of the variables $\left(\phi, \psi, \Psi_{2}, \Psi_{3}\right)$ is much coarser than the characteristic length scale of the morphology. Hence, we also intend to do similarly for the type of multiphase model. We here consider a model that, simultaneously in each volume element, allows us to follow the mass densities and internal energy densities of the phases (solid $s$, liquid $l$ ), as well as of the interface $(i)$, i.e., $\left(\rho_{s}, \rho_{l}, \rho_{i}\right)$ and $\left(\epsilon_{s}, \epsilon_{l}, \epsilon_{i}\right)$, respectively. Knowing the thermodynamic potential (namely the entropy) of the single phases, this choice of variables also keeps track of the temperatures, pressures, and chemical potentials of the single phases. Additionally, the total momentum density $\mathbf{u}=\left(\rho_{s}+\rho_{l}+\rho_{i}\right) \mathbf{v}$ (where $\mathbf{v}$ is the velocity field) is included for situations of flow. A comprehensive introduction to this type of multiphase model can be found in the books of Ishii [10] and Drew and Passman [11]. The approximation of choosing a single velocity field is justified either by neglecting external forces and relative diffusion of the phases or by assuming infinitely high interfacial friction between the two phases [10, 12]. If the densities are defined per unit volume of the whole system rather than per volume of the particular 
phase, the evolution equations can be written in the form (for $k=s, l, i)[10,13]$

$$
\begin{aligned}
\partial_{t} \rho_{k}+\nabla \cdot\left(\mathbf{v} \rho_{k}\right) & =\sum_{\mu=0}^{3} a_{\rho_{k}, \mu} \Upsilon_{\mu}(\mathbf{x}) \\
\partial_{t} \mathbf{u}+\nabla \cdot(\mathbf{v u}) & =\nabla \cdot \sum_{k=s, l, i}\left(\tau_{k}-p_{k} \mathbf{1}\right)+\sum_{\mu=0}^{3} a_{\mathbf{u}, \mu} \Upsilon_{\mu}(\mathbf{x}) \\
\partial_{t} \epsilon_{k}+\nabla \cdot\left(\mathbf{v} \epsilon_{k}\right) & =-p_{k}(\nabla \cdot \mathbf{v})+\tau_{k}:(\nabla \mathbf{v})-\nabla \cdot \mathbf{q}_{k}+\epsilon_{k}^{q}+\sum_{\mu=0}^{3} a_{\epsilon_{k}, \mu} \Upsilon_{\mu}(\mathbf{x})
\end{aligned}
$$

when neglecting turbulent contributions. Here and in the remainder of the manuscript, the arguments $\mathbf{r}$ and $t$ are omitted for convenience. Let us briefly comment on the various contributions (for more details the reader is referred to $[10,12])$. The apparent pressures $p_{k}$ are related to the real pressure in the phases $\tilde{p}_{k}$, the latter resulting from the thermodynamic potential of the respective phase. Using $\phi_{s} \equiv \phi$ and $\phi_{l} \equiv 1-\phi$, we have under specific circumstances $p_{s}=\phi_{s} \tilde{p}_{s}, p_{l}=\phi_{l} \tilde{p}_{l}$, and $p_{i}=\frac{2}{3} \psi \tilde{p}_{i}$ [13], but as discussed below and in detail in [14] different relations need to be considered. Neglecting elastic effects, the stresses $\tau_{k}$ and heat fluxes $\mathbf{q}_{k}$ are expressed as

$$
\begin{array}{rlr}
\tau_{k} & =\phi_{k} \eta_{k}\left((\nabla \mathbf{v})+(\nabla \mathbf{v})^{T}\right)+2 \phi_{k} \hat{\kappa}_{k}(\nabla \cdot \mathbf{v}) \mathbf{1} & (k=s, l), \\
\mathbf{q}_{k} & =-\phi_{k} \lambda_{k} \cdot\left(\nabla T_{k}\right) & (k=s, l), \\
\tau_{i} & =\mathbf{0}, & \\
\mathbf{q}_{i} & =\mathbf{0}, &
\end{array}
$$

with the effective viscosities $\eta_{k}$, the effective dilational viscosities $\kappa_{k}, \hat{\kappa}_{k}=\kappa_{k} / 2-\eta_{k} / 3$, and the (generally anisotropic) effective heat conduction tensors $\lambda_{k}$ (for details see [12]). Analogous to the pressures $\tilde{p}_{k}$, the temperatures $T_{k}$ result from the thermodynamic potential of the respective phase. The relations (1.10a)-(1.10d) for the stresses and heat fluxes describe transport on a macroscopic scale. For example, the effective heat conductivity of the solid phase is zero if isolated crystals are surrounded by melt and in the absence of collisions, because in that scenario no heat can be transferred within the solid phase on a macroscopic scale [12]. A detailed discussion of the different phenomena included in the effective viscosity $\eta_{\alpha}$ is given, e.g., in [15].

In contrast to most terms discussed above, the contributions $\epsilon_{k}^{q}$ in the equations (1.9c) are specific for multiphase flow. They represent the transport of heat across the interface on a length scale fine enough to resolve the exact geometry of the solid-liquid interface. It is assumed that this term is proportional to the amount of interface per unit volume $\psi$ and that it is driven by temperature differences, i.e., [10, 12]

$$
\begin{aligned}
\epsilon_{k}^{q} & =\psi \frac{\lambda_{k}^{(0)}}{l_{k}^{(0)}}\left(T_{i}-T_{k}\right) \quad(k=s, l), \\
\epsilon_{i}^{q} & =-\left(\epsilon_{s}^{q}-\epsilon_{l}^{q}\right),
\end{aligned}
$$

where the last equation results from energy conservation [13]. In contrast to the effective heat conductivity used in (1.10b), here the microscopic heat conductivity $\lambda_{k}^{(0)}$ is required. The characteristic microscopic heat conduction length $l_{k}^{(0)}$ is a measure for the decay of the temperature in phase $k$ from its value at the interface to its bulk 
value [12], the detailed physics of which is not resolved by the more coarse-grained model discussed here.

The phase change contributions in (1.9a)-(1.9c) are captured by the terms proportional to $\Upsilon_{\mu}$ according to the representation (1.8). The specific choice of the coefficients $\mathbf{a}_{\mu} \equiv\left(a_{\rho_{s}, \mu}, a_{\rho_{l} \mu}, a_{\rho_{i}, \mu}, a_{\mathbf{u}, \mu}, a_{\epsilon_{s}, \mu}, a_{\epsilon_{l} \mu}, a_{\epsilon_{i}, \mu}\right)$ must comply with total energy, mass, and momentum conservation. In the present study we choose [13]

$(1.12) \mathbf{a}_{0}=\left(\begin{array}{c}\hat{\rho} \\ -\hat{\rho} \\ 0 \\ \mathbf{0} \\ \epsilon_{s}^{\Gamma} \\ -\epsilon_{l}^{\Gamma} \\ -\left(\epsilon_{s}^{\Gamma}-\epsilon_{l}^{\Gamma}\right)\end{array}\right), \quad \mathbf{a}_{1}=\left(\begin{array}{c}0 \\ 0 \\ 0 \\ \mathbf{0} \\ 0 \\ 0 \\ 0\end{array}\right), \quad \mathbf{a}_{2}=\left(\begin{array}{l}0 \\ 0 \\ 0 \\ \mathbf{0} \\ 0 \\ 0 \\ 0\end{array}\right), \quad \mathbf{a}_{3}=\left(\begin{array}{l}0 \\ 0 \\ 0 \\ \mathbf{0} \\ 0 \\ 0 \\ 0\end{array}\right)$

The coefficient $\hat{\rho}$ associated with mass transfer is often chosen as the solid mass density $\tilde{\rho}_{s}[16,17]$. As far as specific expressions for the coefficients $\epsilon_{s}^{\Gamma}$ and $\epsilon_{l}^{\Gamma}$ are concerned, the reader is referred to $[10,12,15,18]$.

We mention that by choosing the multiphase model described above, the description of flow-induced crystallization of polymers is not possible. For the latter, memory effects of the material need to be included to monitor the distortion of the chain configuration, either by adding elastic contributions to the stresses or even by introducing an additional structural variable for the chain configuration.

1.3. Methodology: GENERIC framework of nonequilibrium thermodynamics. Recently, a general equation for the nonequilibrium reversible-irreversible coupling (GENERIC) has been developed for describing nonequilibrium systems [19, 20]. When trying to formulate a model in the GENERIC framework, the first step is to choose the variables that describe the system. Similar to the procedure in equilibrium thermodynamics, the choice of variables must be such that they are independent and sufficient to capture the essential physics. Such a set of variables shall here be denoted by $x$. Note that $x$ may have both discrete indices as well as continuous indices (for field variables). According to GENERIC, the time evolution of the variables $x$ can be written in the form

$$
\frac{d x}{d t}=L(x) \frac{\delta E}{\delta x}+M(x) \frac{\delta S}{\delta x},
$$

where the two generators $E$ and $S$ are the total energy and entropy functionals in terms of the state variables $x$, and $L$ and $M$ are certain matrices (operators). The matrix multiplications imply not only summations over discrete indices but may also include integration over continuous variables, and $\delta / \delta x$ typically implies functional rather than partial derivatives (for more details see $[19,20]$ ). The GENERIC structure also imposes certain conditions on the building blocks in (1.13). First, (1.13) is supplemented by the degeneracy requirements

$$
\begin{aligned}
& L(x) \frac{\delta S}{\delta x}=0, \\
& M(x) \frac{\delta E}{\delta x}=0 .
\end{aligned}
$$

The requirement that the (functional) derivative of the entropy lies in the null space of $L$ represents the reversible nature of $L$. Hence, the functional form of $S$ and $L$ 
are constrained such that the entropy is not affected by the operator generating the reversible dynamics, i.e., by $L$. On the other hand, the requirement that the functional derivative lies in the null space of $M$ manifests that the total energy is not altered by the $M$-contribution to the dynamics. In addition to these degeneracy requirements, $L$ must be antisymmetric and fulfill the Jacobi identity, whereas $M$ needs to be positivesemidefinite and Onsager-Casimir symmetric. As a result of all these conditions one may easily show that the GENERIC equation (1.13) implies both the conservation of total energy, as well as a nonnegative entropy production. The two contributions to the time evolution of $x$ generated by the total energy $E$ and the entropy $S$ in (1.13) are called the reversible and irreversible contributions, respectively.

Both the complementary degeneracy requirements and the symmetry properties are essential for formulating proper $L$ - and $M$-matrices when modeling concrete nonequilibrium problems. The list of systems, which have already been expressed in the GENERIC form, includes classical hydrodynamics, polymer kinetic theory (including hydrodynamic interaction, rigid constraints, reptation models, and polymer heat conductivity), chemical reactions, Boltzmann's kinetic equation, the Doi-Ohta model, and multiphase models with phase change [13, 14]. These various applications have shown that the two-generator idea and the degeneracy requirements have strong implications. In order to justify this approach, these elements of GENERIC, originally discovered by empirical observations, have been derived by projection operator formalisms [21, 22], which strongly encourage using the GENERIC formalism.

1.4. Thermodynamic contributions to nucleation and growth. Now, the previously developed results [13] for the phase change contributions in the multiphase model described above shall be illustrated, as well as the associated fundamental problem.

In the unification of the multiphase model (1.9a)-(1.9c) (m) with the morphological model (1.8) within the GENERIC framework, the set of variables to describe the system was given by [13]

$$
\mathbf{x}=\mathbf{x}^{\mathrm{m}} \oplus \mathbf{\Phi}=\left(\rho_{s}, \rho_{l}, \rho_{i}, \mathbf{u}, \epsilon_{s}, \epsilon_{l}, \epsilon_{i}, \phi, \psi, \Psi_{2}, \Psi_{3}\right),
$$

with

$$
\mathbf{x}^{\mathrm{m}} \equiv\left(\rho_{s}, \rho_{l}, \rho_{i}, \mathbf{u}, \epsilon_{s}, \epsilon_{l}, \epsilon_{i},\right)
$$

Natural expressions for the energy functional $E$ and for the entropy functional $S$ were obtained by the local equilibrium assumption. If the thermodynamics of the solid, the liquid, and the interface is characterized by $s_{s}, s_{l}$, and $s_{i}$, respectively, the generating functionals $E$ and $S$ read

$$
\begin{aligned}
& E[\mathbf{x}]=\int\left(\frac{1}{2} \frac{\mathbf{u}^{2}}{\rho_{s}+\rho_{l}+\rho_{i}}+\epsilon_{s}+\epsilon_{l}+\epsilon_{i}\right) d^{3} r, \\
& S[\mathbf{x}]=\int\left(\phi s_{s}\left(\tilde{\rho}_{s}, \tilde{\epsilon}_{s}\right)+(1-\phi) s_{l}\left(\tilde{\rho}_{l}, \tilde{\epsilon}_{l}\right)+\psi s_{i}\left(\tilde{\rho}_{i}, \tilde{\epsilon}_{i}\right)\right) d^{3} r,
\end{aligned}
$$

where the intrinsic quantities denoted by " $\sim$ " for the indices $(s, l)$ are densities per volume of the respective phase (and not with respect to the total volume of the volume element) and for the index $i$ are densities per amount of interface. They can be expressed in terms of the corresponding apparent variables contained in $\mathbf{x}$ as

$$
\begin{aligned}
& \tilde{\rho}_{s}=\frac{\rho_{s}}{\phi}, \quad \tilde{\rho}_{l}=\frac{\rho_{l}}{1-\phi}, \quad \tilde{\rho}_{i}=\frac{\rho_{i}}{\psi}, \\
& \tilde{\epsilon}_{s}=\frac{\epsilon_{s}}{\phi}, \quad \tilde{\epsilon}_{l}=\frac{\epsilon_{l}}{1-\phi}, \quad \tilde{\epsilon}_{i}=\frac{\epsilon_{i}}{\psi} .
\end{aligned}
$$


We mention that the above entropy expression (1.18b) accounts neither for the configurational entropy of the interface nor for the relative arrangements of the dispersed component. Using the following relations for the temperatures, the chemical potentials per unit mass, and the pressures (for $k=s, l, i$ ),

$$
\begin{aligned}
T_{k} & =\left(\frac{\partial s_{k}\left(\tilde{\rho}_{k}, \tilde{\epsilon}_{k}\right)}{\partial \tilde{\epsilon}_{k}}\right)^{-1}, \\
\frac{\mu_{k}}{T_{k}} & =-\frac{\partial s_{k}\left(\tilde{\rho}_{k}, \tilde{\epsilon}_{k}\right)}{\partial \tilde{\rho}_{k}}, \\
\frac{\tilde{p}_{k}}{T_{k}} & =s_{k}\left(\tilde{\rho}_{k}, \tilde{\epsilon}_{k}\right)-\tilde{\epsilon}_{k} \frac{\partial s_{k}\left(\tilde{\rho}_{k}, \tilde{\epsilon}_{k}\right)}{\partial \tilde{\epsilon}_{k}}-\tilde{\rho}_{k} \frac{\partial s_{k}\left(\tilde{\rho}_{k}, \tilde{\epsilon}_{k}\right)}{\partial \tilde{\rho}_{k}},
\end{aligned}
$$

the functional derivatives $\delta E[\mathbf{x}] / \delta \mathbf{x}$ and $\delta S[\mathbf{x}] / \delta \mathbf{x}$, needed for the GENERIC formulation, can be related to the intensive thermodynamic properties of the phases.

Formulating the phase change contributions in the dynamic equation amounts to finding a positive semidefinite, Onsager-Casimir symmetric operator $\mathbf{M}^{\mathrm{pc}}$ that fulfills the degeneracy requirement (1.15). First, it has been found in [13] that the relevant quantities driving the phase change are not directly the functional derivatives of $E$ and $S$ but rather their combinations with the coefficients (1.12),

$$
\begin{array}{ll}
\Lambda_{\mu}^{(E)}:=\mathbf{a}_{\mu} \cdot \frac{\delta E}{\delta \mathbf{x}^{\mathrm{m}}}+\frac{\delta E}{\delta \Phi_{\mu}} & (\mu=0,1,2,3), \\
\Lambda_{\mu}^{(S)}:=\mathbf{a}_{\mu} \cdot \frac{\delta S}{\delta \mathbf{x}^{\mathrm{m}}}+\frac{\delta S}{\delta \Phi_{\mu}} & (\mu=0,1,2,3) .
\end{array}
$$

For the energy and entropy functionals specified in (1.18a), (1.18b), we have

$$
\frac{\delta E}{\delta \mathbf{x}}=\left(\begin{array}{c}
-\frac{1}{2} \mathbf{v}^{2} \\
-\frac{1}{2} \mathbf{v}^{2} \\
-\frac{1}{2} \mathbf{v}^{2} \\
\mathbf{v} \\
1 \\
1 \\
1 \\
0 \\
0 \\
0 \\
0
\end{array}\right), \quad \quad \frac{\delta S}{\delta \mathbf{x}}=\left(\begin{array}{c}
-\frac{\mu_{s}}{T_{s}} \\
-\frac{\mu_{l}}{T_{l}} \\
-\frac{\mu_{i}}{T_{i}} \\
0 \\
\frac{1}{T_{s}} \\
\frac{1}{T_{l}} \\
\frac{1}{T_{i}} \\
\frac{\tilde{p}_{s}}{T_{s}}-\frac{\tilde{p}_{l}}{T_{l}} \\
-\frac{\sigma}{T_{i}} \\
0 \\
0
\end{array}\right)
$$

and hence

(1.23a) $\boldsymbol{\Lambda}^{(E)}=\mathbf{0}$,

$$
\Lambda^{(S)}=\left(\begin{array}{c}
\epsilon_{s}^{\Gamma}\left(\frac{1}{T_{s}}-\frac{1}{T_{i}}\right)+\epsilon_{l}^{\Gamma}\left(\frac{1}{T_{i}}-\frac{1}{T_{l}}\right)-\hat{\rho}\left(\frac{\mu_{s}}{T_{s}}-\frac{\mu_{l}}{T_{l}}\right)+\left(\frac{\tilde{p}_{s}}{T_{s}}-\frac{\tilde{p}_{l}}{T_{l}}\right) \\
-\frac{\sigma}{T_{i}} \\
0 \\
0
\end{array}\right)
$$

if the surface tension $\sigma$ is given by $\sigma=-\tilde{p}_{i}$. The absence of the interfacial chemical potential $\mu_{i}$ is due to the fact that the interface does not participate in the mass 
exchange, as the choice of $\mathbf{a}_{1}$ in (1.12) indicates. The task of finding the operator $\mathbf{M}^{\mathrm{pc}}$ is then reduced to finding a positive semidefinite matrix $A_{\mu \nu}$, with which (see [13] for more details)

$$
\left.\partial_{t} \Phi_{\mu}\right|_{\mathrm{pc}}=\sum_{\nu=0}^{3} A_{\mu \nu}(\mathbf{x}) \Lambda_{\nu}^{(S)}(\mathbf{x}) \quad(\mu=0,1,2,3) .
$$

The corresponding matrix $\mathbf{M}^{\mathrm{pc}}$ can be written as

$$
\mathbf{M}^{\mathrm{pc}}=\left(\begin{array}{cc}
M_{x_{\alpha}}^{\mathrm{pc}}, x_{\beta}^{\mathrm{m}} & M_{x_{\alpha}^{\mathrm{m}}, \Phi_{\gamma}}^{\mathrm{pc}} \\
M_{\Phi_{\gamma}, x_{\alpha}^{\mathrm{m}}}^{\mathrm{pc}} & M_{f \Phi_{\gamma}, \Phi_{\delta}}^{\mathrm{pc}}
\end{array}\right)
$$

with

$$
\begin{aligned}
M_{x_{\alpha}^{\mathrm{m}}, x_{\beta}^{\mathrm{m}}}^{\mathrm{pc}} & =A_{\mu \nu} a_{x_{\alpha}^{\mathrm{m}} \mu} a_{x_{\beta}^{\mathrm{m}} \nu}, \\
M_{x_{\alpha}^{\mathrm{m}}, \Phi_{\gamma}}^{\mathrm{pc}} & =A_{\mu \gamma} a_{x_{\alpha}^{\mathrm{m}} \mu}, \\
M_{\Phi_{\gamma}, x_{\alpha}^{\mathrm{m}}}^{\mathrm{pc}} & =A_{\gamma \nu} a_{x_{\alpha}^{\mathrm{m}} \nu}, \\
M_{\Phi_{\gamma}, \Phi_{\gamma^{\prime}}}^{\mathrm{pc}} & =A_{\gamma \gamma^{\prime}},
\end{aligned}
$$

which shall be used for comparison in sections below. If the equations (1.24), which are (1.8) with the thermodynamic background of the multiphase model, are matched with the dynamic equations (1.6a)-(1.6d), one finds the most intriguing result for the growth and nucleation rate [13]

$$
\begin{aligned}
G & =\left(\Lambda_{1}^{(S)}+\frac{\mathcal{L}}{\psi} \Lambda_{2}^{(S)}\right) \mathcal{R}, \\
8 \pi \alpha & =\left(\Lambda_{1}^{(S)}+\frac{\mathcal{L}}{\psi} \Lambda_{2}^{(S)}\right) \mathcal{R} \mathcal{Q}=G \mathcal{Q} .
\end{aligned}
$$

In order to have a well-defined operator $M^{\mathrm{pc}}$, both functions $\mathcal{R}\left[\mathrm{m}^{4} \mathrm{~K} \mathrm{~s}^{-1} \mathrm{~J}^{-1}\right]$ and $\mathcal{Q}\left[\mathrm{m}^{-4}\right]$ must be well-defined functions of $\mathbf{x}$, whereas the positivity of $M^{\mathrm{pc}}$ requires $\mathcal{R} \geq 0$. This determines the structure of the matrix $A_{\mu \nu}$.

The result given in (1.27a), (1.27b) has four prominent features. First, it demonstrates the intimate relation between the phase change coefficients in (1.9a)-(1.9c), on one hand, and the thermodynamic driving force for crystallization through (1.21b) on the other hand. In particular, it allows us to discern between crystallization and melting conditions. However, the kinetic contributions, i.e., the prefactors, are not restricted by the current treatment. Second, in zero-growth conditions $(G=0)$, also the nucleation rate must vanish since $\mathcal{Q}$ is a well-defined function without poles. Third, the no-crystallization condition is size-dependent due to the prefactor $\mathcal{L} / \psi$ to the surface tension contribution $\Lambda_{2}^{(S)}$. The fourth property of $(1.27 \mathrm{a}),(1.27 \mathrm{~b})$ is a problematic one, as it concerns a shortcoming of the model in the early stages of crystallization. The surface tension contribution both in $G$ and in $\alpha$ is dominating if the ratio $\mathcal{L} / \psi\left[\mathrm{m}^{-1}\right]$, of the order of the inverse crystal length scale, is sufficiently large. This directly leads us to formulating the main goal of the present manuscript.

1.5. Goal. It is known in classical nucleation theory (see, e.g., $[23,24,25])$ that the presence of surface tension amounts to an energy barrier, which spontaneously forming nuclei have to overcome before they can continue to grow under the influence of the bulk contributions (usually in the Helmholtz free energy difference). The 
problem of the model developed in [13] in the early stages of crystallization is an image of this energy barrier, appropriately recast to the formulation in which the microstructure is described by the variables $\boldsymbol{\Phi}=\left(\phi, \psi, \Psi_{2}, \Psi_{3}\right)$. According to current understanding of nucleation, fluctuations enable the formation of so-called supercritical nuclei. Therefore, the restriction to modeling purely deterministic morphological variables must be released if the situation of nucleation shall be clarified. Furthermore, a distinction between subcritical and supercritical nuclei on grounds of the variables in the model must be possible. As a result of these comments, the morphological description in terms of $\boldsymbol{\Phi}$ is replaced by a distribution function of cluster sizes. In the following, the general term "clusters" is intentionally used instead of "crystallites," since as will become clear below, the model will apart from crystallites also encompass unstable and stable nuclei, all of them being denoted as clusters. Restricting the modeling to spherical clusters, we choose the distribution function $f(\mathbf{r}, R, t)$ as relevant variable. It denotes the number of clusters of radius in the interval $[R, R+d R$ [ in the volume element $d r^{3}$ at position $\mathbf{r}$ at time $t$. In general, the relation between the cluster size distribution $f(R)$ and $\boldsymbol{\Phi}$ (in particular $\phi$ and $\psi$ ) depends on the spatial correlation of the clusters. However, the Boolean grain model helps in this respect if one assumes that nuclei (and subsequently the crystals) are formed randomly in the volume element under consideration. If we define

$$
\begin{aligned}
\Psi_{\mu} & :=\frac{8 \pi}{(3-\mu) !} \int_{0}^{\infty} R^{(3-\mu)} f(R) d R \\
& \equiv \frac{8 \pi}{(3-\mu) !}\left\langle R^{(3-\mu)}\right\rangle_{f} \quad(\mu=0,1,2,3),
\end{aligned}
$$

in accordance with (1.2), the expressions for $\phi$ and $\psi$ in terms of $f(R)$ then follow assuming the Boolean grain model through (1.4a), (1.4b), hence completing the projection $f(R) \rightarrow \boldsymbol{\Phi}$. So, the new model we envisage, first, goes beyond capturing the zeroth to third moments of $f(R)$ and, second, offers the possibility of including diffusive effects for the nucleation by means of formulating a diffusion equation for the distribution function.

\section{Evolution of cluster size distribution during solidification.}

2.1. Set of variables and generating functionals. Based on the above motivation, the thermodynamic states of the competing phases and of the interface shall again be described by $\mathbf{x}^{\mathrm{m}}$ given in (1.17), whereas the morphology shall be monitored through the cluster size distribution function $f(R)$, with $R$ the cluster radius in the interval $[R, R+d R[$. Hence, the set of fundamental variables for the GENERIC description is

$$
\mathbf{x}^{\prime} \equiv \mathbf{x}^{\mathrm{m}} \oplus f(R)=\left(\rho_{s}, \rho_{l}, \rho_{i}, \mathbf{u}, \epsilon_{s}, \epsilon_{l}, \epsilon_{i}, f(R)\right) .
$$

Subsequently, the GENERIC model with the variables (1.16) is called " $\Phi$-model," and the one developed with $(2.1)$ is denoted by " $f$-model." The normalization for the distribution function $f(R)$ is given by

$$
n=\int_{0}^{\infty} f(R) d R=\langle 1\rangle_{f},
$$

where $n$ denotes the number of clusters per unit volume. The major difference to the previous model being the altered morphological description, $f(R)$ instead of $\boldsymbol{\Phi}$, the 
generating functionals for the new model in terms of $\mathbf{x}^{\prime}$ are basically given by (1.18a), (1.18b), where the variables $\boldsymbol{\Phi}$ are now functionals of the distribution $f(R)$. Furthermore, the distribution $f(R)$ brings about an additional entropy contribution. As the fundamental process of phase transition consists of adding/removing discrete quantities of volumes, i.e., "molecules," the distribution $\tilde{f}(v)$ of cluster volumes $v=\frac{4 \pi}{3} R^{3}$ is the more fundamental variable than the distribution of radii, $f(R)$, in agreement with classical nucleation theory $[23,25,26]$. The proper entropy expression due to the cluster size distribution is hence given by the well-known Boltzmann-Gibbs entropy in terms of $\tilde{f}(v)$. If this is then expressed in terms of $f(R)$ (see the appendix for details), the generating functionals are finally given by

$$
\begin{aligned}
& E\left(\mathbf{x}^{\prime}\right)=\left.E\left(\mathbf{x}^{\mathrm{m}}, \mathbf{\Phi}\right)\right|_{\mathbf{\Phi}=\mathbf{\Phi}[f(R)]}, \\
& S\left(\mathbf{x}^{\prime}\right)=\left.S\left(\mathbf{x}^{\mathrm{m}}, \mathbf{\Phi}\right)\right|_{\mathbf{\Phi}=\mathbf{\Phi}[f(R)]}-k_{\mathrm{B}} \int d^{3} r \int_{0}^{\infty} d R f(R) \ln \left(\frac{f(R)}{4 \pi R^{2}}\right),
\end{aligned}
$$

where the functions $E\left(\mathbf{x}^{\mathrm{m}}, \mathbf{\Phi}\right)$ and $S\left(\mathrm{x}^{\mathrm{m}}, \mathbf{\Phi}\right)$ are the ones specified in (1.18a), (1.18b). We point out that although the entropy expression is slightly more complicated if formulated in terms of $f(R)$ than in terms of $\tilde{f}(v)$, the distribution of radii $f(R)$ is more convenient for establishing the relation to the coarse-grained variables $\boldsymbol{\Psi}$ (1.28) or $\boldsymbol{\Phi}$ (1.4a)-(1.5), respectively. The functional derivatives of $E\left(\mathbf{x}^{\prime}\right)$ and $S\left(\mathbf{x}^{\prime}\right)$ with respect to $\mathbf{x}^{\prime}$ then read

$\frac{\delta E}{\delta \mathbf{x}^{\prime}}=\left(\begin{array}{c}-\frac{1}{2} \mathbf{v}^{2} \\ -\frac{1}{2} \mathbf{v}^{2} \\ -\frac{1}{2} \mathbf{v}^{2} \\ \mathbf{v} \\ 1 \\ 1 \\ 1 \\ 0\end{array}\right), \quad \frac{\delta S}{\delta \mathbf{x}^{\prime}}=\left(\begin{array}{c}-\frac{\mu_{s}}{T_{s}} \\ -\frac{\mu_{l}}{T_{l}} \\ -\frac{\mu_{i}}{T_{i}} \\ 0 \\ \frac{1}{T_{s}} \\ \frac{1}{T_{l}} \\ \frac{1}{T_{i}} \\ \left(\frac{\tilde{p}_{s}}{T_{s}}-\frac{\tilde{p}_{l}}{T_{l}}\right) \frac{\delta \phi}{\delta f(R)}-\frac{\sigma}{T_{i}} \frac{\delta \psi}{\delta f(R)}-k_{\mathrm{B}}\left(\ln \left(\frac{f(R)}{4 \pi R^{2}}\right)+1\right)\end{array}\right)$.

2.2. Reversible dynamics. We now discuss the reversible contributions, i.e., the determination of the Poisson operator $L$. In the present discussion, the reversible contributions are convective in nature. In analogy to the $\boldsymbol{\Phi}$-model described in [13], we propose the Poisson operator corresponding to the following Poisson bracket:

$$
\begin{aligned}
\{A, B\} \equiv & \left\langle\frac{\delta A}{\delta \mathbf{x}^{\prime}}, L \frac{\delta B}{\delta \mathbf{x}^{\prime}}\right\rangle \\
= & -\sum_{k=s, l, i} \int \rho_{k}\left(\frac{\delta A}{\delta u_{m}} \frac{\partial}{\partial r_{m}} \frac{\delta B}{\delta \rho_{k}}-\frac{\delta B}{\delta u_{m}} \frac{\partial}{\partial r_{m}} \frac{\delta A}{\delta \rho_{k}}\right) d^{3} r \\
& -\int u_{n}\left(\frac{\delta A}{\delta u_{m}} \frac{\partial}{\partial r_{m}} \frac{\delta B}{\delta u_{n}}-\frac{\delta B}{\delta u_{m}} \frac{\partial}{\partial r_{m}} \frac{\delta A}{\delta u_{n}}\right) d^{3} r \\
& -\sum_{k=s, l, i} \int \epsilon_{k}\left(\frac{\delta A}{\delta u_{m}} \frac{\partial}{\partial r_{m}} \frac{\delta B}{\delta \epsilon_{k}}-\frac{\delta B}{\delta u_{m}} \frac{\partial}{\partial r_{m}} \frac{\delta A}{\delta \epsilon_{k}}\right) d^{3} r \\
& -\sum_{k=s, l, i} \int p_{k}\left(\mathbf{x}^{\prime}\right)\left(\frac{\delta A}{\delta \epsilon_{k}} \frac{\partial}{\partial r_{m}} \frac{\delta B}{\delta u_{m}}-\frac{\delta B}{\delta \epsilon_{k}} \frac{\partial}{\partial r_{m}} \frac{\delta A}{\delta u_{m}}\right) d^{3} r
\end{aligned}
$$




$$
\begin{aligned}
& +\iint_{0}^{\infty} \frac{\delta A}{\delta u_{m}} L_{u_{m}, f}\left(R^{\prime}\right) \frac{\delta B}{\delta f\left(R^{\prime}\right)} d R^{\prime} d^{3} r \\
& +\iint_{0}^{\infty} \frac{\delta A}{\delta f(R)} L_{f, u_{m}}(R) \frac{\delta B}{\delta u_{m}} d R d^{3} r
\end{aligned}
$$

where $A$ and $B$ denote two test functionals. The index $k$ sums over the solid $(s)$, liquid $(l)$, and interface $(i)$ properties, whereas the indices $m$ and $n$ denote Cartesian components of vectors and the Einstein summation convention is employed. The GENERIC properties of the above Poisson operator can be discussed only after specifying the pressure expressions $p_{k}\left(\mathbf{x}^{\prime}\right)$, as well as the elements $L_{u_{m}, f}\left(R^{\prime}\right)$ and $L_{f, u_{m}}(R)$, which represent the convection of $f(R)$. The $\left(R, R^{\prime}\right)$-notation is analogous to the one for discrete indices. Functions multiplied from the left to an element of the Poisson operator carry the argument $R$, whereas functions multiplied from the right have argument $R^{\prime}$, and an integration is performed over every doubly occurring continuous argument. We note that in principle a similar procedure is appropriate for the argument $\mathbf{r}$ but can be eliminated in the present case as shown also in [20].

Let us now concentrate on the convective contributions for the distribution function $f(R)$. Since spatially homogeneous flows shall have no effect on $R$ and since $R$ lacks orientational information, its change due to flow must be proportional to the scalar $\nabla \cdot \mathbf{v}$ if we omit nonlinear effects in $\nabla \mathbf{v}$. If the interface between the two phases transforms affinely under flow, in particular in expansion flow, the radius of a certain cluster evolves as

$$
\dot{R}=\frac{R}{3}(\nabla \cdot \mathbf{v})
$$

However, if the phase of which the clusters consists is incompressible, we have

$$
\dot{R}=0 \text {. }
$$

The flow contribution to the evolution equation can hence be summarized by

$$
\begin{aligned}
\left.\partial_{t} f(R)\right|_{\text {flow }} & =-\nabla f(R) \mathbf{v}-\partial_{R} \dot{R} f(R) \\
& =-\nabla f(R) \mathbf{v}-\partial_{R} f(R) \Theta_{0} \frac{R}{3}(\nabla \cdot \mathbf{v}),
\end{aligned}
$$

where $\Theta_{0}$ is a constant, in particular $\Theta_{0}=1$ for affinely deformed interfaces, and $\Theta_{0}=0$ for incompressible clusters. Nonconstant choices for $\Theta_{0}$ shall not be discussed here. The additional first term in (2.9) is motivated by the fact that the number density $n(2.2)$ should be a scalar density. Considering the functional derivatives of the energy (2.5) and the occurrence of reversible contributions in the GENERIC (1.13), equation (2.9) can be cast into the following elements of the Poisson operator $L:$

$$
\begin{aligned}
L_{f, \mathbf{u}}(R) & =-\nabla f(R)-\Theta_{0} \partial_{R} f(R) \frac{R}{3} \nabla, \\
L_{\mathbf{u}, f}\left(R^{\prime}\right) & =-f\left(R^{\prime}\right) \nabla+\Theta_{0} \nabla f\left(R^{\prime}\right) \frac{R^{\prime}}{3} \partial_{R^{\prime}},
\end{aligned}
$$

where $L_{\mathbf{u}, f}$ is given through $L_{f, \mathbf{u}}$ by means of the antisymmetry requirement on $L$ with respect to the scalar product

$$
\langle A, B\rangle:=\iint_{0}^{\infty} A(\mathbf{r}, R) B(\mathbf{r}, R) d R d^{3} r .
$$


The connection to the convection mechanisms of the variables $\boldsymbol{\Phi}=\left(\phi, \psi, \Psi_{2}, \Psi_{3}\right)$ can be established from the ones of $\Psi_{\mu}(\mu=0,1,2,3)$, for which we find from (2.10a), (2.10b) with (2.5) for the reversible contributions according to the GENERIC (1.13)

$$
\left.\partial_{t} \Psi_{\mu}\right|_{\text {flow }}=-\nabla \cdot\left(\Psi_{\mu} \mathbf{v}\right)+\Theta_{0} \frac{3-\mu}{3}(\nabla \cdot \mathbf{v}) \Psi_{\mu},
$$

where the boundary terms are assumed to vanish. The convection mechanism for $\phi$ and $\psi$ for the Boolean grain model can be determined using (1.4a) and (1.4b), respectively. In particular, the resulting convective contributions to $\boldsymbol{\Phi}$ are closed expressions in terms of $\boldsymbol{\Phi}$. In other words, the functions $\Omega_{\mu}(\mathbf{x})(\mu=0,1,2,3)$, as defined through

$$
\left.\partial_{t} \Phi_{\mu}\right|_{\text {flow }}=-\nabla \cdot\left(\Phi_{\mu} \mathbf{v}\right)-\Omega_{\mu}(\nabla \cdot \mathbf{v})
$$

and studied in detail in [14], can be determined for given $L_{f, \mathbf{u}}\left(R, R^{\prime}\right)$. It must be mentioned that, e.g., scalar density-like transformation behavior, $\Theta_{0}=0$, is not transferred from the moments $\left\langle R^{k}\right\rangle$ to the Minkowski functionals $\boldsymbol{\Phi}$ due to the nonlinearity of their relation. In [14], the pressures $p_{k}$ were found to be related to the functions $\Omega_{\mu}$ as $p_{s}=-\Omega_{0} \tilde{p}_{s}, p_{l}=\left(1+\Omega_{0}\right) \tilde{p}_{l}$, and $p_{s}=-\Omega_{1} \tilde{p}_{i}\left(\tilde{p}_{k}\right.$ being given by $(1.20 \mathrm{c})$ ), which resulted in fulfilling the degeneracy requirement (1.14), as well as the Jacobi identity, as long as the functions $\Omega_{\mu}(\mu=0,1,2,3)$ did not depend on the momentum density, i.e., $\Omega_{\mu}=\Omega_{\mu}(\mathbf{x} \backslash \mathbf{u})$.

In the present study, in which we use $f(R)$ instead of $\boldsymbol{\Phi}$, we find that the degeneracy requirement (1.14) and the Jacobi identity are satisfied for

$$
\begin{aligned}
p_{s} & =\left(\phi-\left(1-\Theta_{0}\right)\left\langle\frac{\delta \phi}{\delta f}\right\rangle_{f}\right) \tilde{p}_{s}+\left(1+\Theta_{0}\right) k_{\mathrm{B}} n T_{s}, \\
p_{l} & =\left((1-\phi)+\left(1-\Theta_{0}\right)\left\langle\frac{\delta \phi}{\delta f}\right\rangle_{f}\right) \tilde{p}_{l}, \\
p_{i} & =\left(\left(1-\frac{\Theta_{0}}{3}\right) \psi-\left(1-\Theta_{0}\right)\left\langle\frac{\delta \psi}{\delta f}\right\rangle_{f}\right) \tilde{p}_{i}
\end{aligned}
$$

for any (constant) value of $\Theta_{0}$. The prefactors of $\tilde{p}_{k}$, which result from the convection of $f(R),(2.10 \mathrm{a}),(2.10 \mathrm{~b})$, and the degeneracy requirement (1.14), exactly correspond to the $\Omega_{\mu}$-contributions examined in [14] for the specific functions $\Omega_{\mu}$ for (2.13) based on (2.12). However, the additional thermal pressure in $p_{s}$ is specific to the distribution function formulation and originates from the additional entropy term in (2.4). First, its prefactor, $1+\Theta_{0}$, is different for an affinely deformed interface $\left(\Theta_{0}=1\right)$ than it is for an incompressible solid phase $\left(\Theta_{0}=0\right)$. Second, we mention that for the sake of a desirable $T_{s}$-dependence, this thermal pressure is to be included in $p_{s}$, instead of in $p_{l}$ for a dependence on $T_{l}$. In conclusion, it can be shown that the Poisson operator given in (2.6) with (2.10a), (2.10b) with the pressure expressions (2.14a), (2.14b), (2.14c) is antisymmetric and fulfills the degeneracy requirement (1.14), as well as the Jacobi identity; i.e., it meets all the requirements of the GENERIC.

2.3. Irreversible dynamics. The irreversible contributions in the dynamic equations (1.9a)-(1.9c) and in the equation for the distribution function $f(R)$ are driven by the entropy gradient in the GENERIC (1.13), the dynamics being specified completely by the metric matrix $M$. The properties of $M$ are additive, and 
hence the metric matrices for physically distinct phenomena can be analyzed separately. Thus, as far as the formulation of the viscous stresses and bulk heat conduction (1.10a)-(1.10d) and interfacial heat transfer (1.11a), (1.11b) are concerned, the reader is referred to [13], where the corresponding $M$-contributions are specified. We may hence concentrate here on the phase change contributions, i.e., on the irreversible contributions to $f(R)$. For later convenience, the metric matrix for phase change is split according to the variables $(2.1)$ as

$$
\mathbf{M}^{\mathrm{pc}}=\left(\begin{array}{cc}
M_{x_{\alpha}^{\mathrm{m}}, x_{\beta}^{\mathrm{m}}}^{\mathrm{pc}} & M_{x_{\alpha}^{\mathrm{m}}, f}^{\mathrm{pc}} \\
M_{f, x_{\alpha}^{\mathrm{m}}}^{\mathrm{pc}} & M_{f, f}^{\mathrm{pc}}
\end{array}\right) .
$$

First, we write down all phase change contributions (subscript "pc") for the multiphase model (m) (1.9a)-(1.9c) with (1.8), using Einstein summation convention,

$$
\begin{aligned}
\left.\partial_{t} x_{\alpha}^{\mathrm{m}}\right|_{\mathrm{pc}} & =\left.a_{x_{\alpha}^{\mathrm{m}}, \mu}\left(\mathbf{x}^{\mathrm{m}}, \boldsymbol{\Phi}[f]\right) \partial_{t} \Phi_{\mu}\right|_{\mathrm{pc}} \\
& =\left.a_{x_{\alpha}^{\mathrm{m}}, \mu}\left(\mathbf{x}^{\mathrm{m}}, \mathbf{\Phi}[f]\right) \int_{0}^{\infty} \frac{\delta \Phi_{\mu}}{\delta f(R)} \partial_{t} f(R)\right|_{\mathrm{pc}} d R \\
& =M_{x_{\alpha}^{\mathrm{m}}, x_{\beta}^{\mathrm{m}}}^{\mathrm{p}} \frac{\delta S}{\delta x_{\beta}^{\mathrm{m}}}+M_{x_{\alpha}^{\mathrm{m}}, f}^{\mathrm{pc}} \frac{\delta S}{\delta f},
\end{aligned}
$$

where the functions $a_{x_{\alpha}^{\mathrm{m}} \mu}$ are given, e.g., in (1.12) and are not to be determined from the following discussion. Now, if we assume that the "phase change" contribution to the equation of $f(R)$ can be captured within the GENERIC, we may write in most generality

$$
\left.\partial_{t} f(R)\right|_{\mathrm{pc}}=M_{f, x_{\beta}^{\mathrm{m}}}^{\mathrm{pc}} \frac{\delta S}{\delta x_{\beta}^{\mathrm{m}}}+M_{f, f}^{\mathrm{pc}} \frac{\delta S}{\delta f} .
$$

Substitution of this expression into (2.16b) and comparison with (2.16c) results in the following relation between the elements of $\mathbf{M}^{\mathrm{pc}}$ :

$$
\begin{array}{r}
a_{x_{\alpha}^{\mathrm{m}}, \mu} \int_{0}^{\infty} \frac{\delta \Phi_{\mu}}{\delta f(R)}\left(M_{f, x_{\beta}^{\mathrm{m}}}^{\mathrm{pc}} \frac{\delta S}{\delta x_{\beta}^{\mathrm{m}}}+M_{f, f}^{\mathrm{pc}} \frac{\delta S}{\delta f}\right) d R \\
=M_{x_{\alpha}^{\mathrm{m}}, x_{\beta}^{\mathrm{m}}}^{\mathrm{pc}} \frac{\delta S}{\delta x_{\beta}^{\mathrm{m}}}+M_{x_{\alpha}^{\mathrm{m}}, f}^{\mathrm{pc}} \frac{\delta S}{\delta f} .
\end{array}
$$

The structure of the relation (2.18) suggests

$$
\begin{aligned}
M_{x_{\alpha}^{\mathrm{m}}, x_{\beta}^{\mathrm{m}}}^{\mathrm{pc}} & =\int_{0}^{\infty} a_{x_{\alpha}^{\mathrm{m}} \mu} \frac{\delta \Phi_{\mu}}{\delta f\left(R^{\prime \prime}\right)}\left(D\left(R^{\prime \prime}\right) a_{x_{\beta}^{\mathrm{m}}} \nu \frac{\delta \Phi_{\nu}}{\delta f\left(R^{\prime \prime}\right)}\right) d R^{\prime \prime}, \\
M_{x_{\alpha}^{\mathrm{m}}, f}^{\mathrm{pc}}\left(R^{\prime}\right) & =a_{x_{\alpha}^{\mathrm{m}} \nu} \frac{\delta \Phi_{\nu}}{\delta f\left(R^{\prime}\right)} D\left(R^{\prime}\right), \\
M_{f, x_{\alpha}^{\mathrm{m}}}^{\mathrm{pc}}(R) & =\left(D(R) a_{x_{\alpha}^{\mathrm{m}} \nu} \frac{\delta \Phi_{\nu}}{\delta f(R)}\right), \\
M_{f, f}^{\mathrm{pc}}\left(R, R^{\prime}\right) & =\delta\left(R-R^{\prime}\right) D\left(R^{\prime}\right),
\end{aligned}
$$

where the $\left(R, R^{\prime}\right)$-notation is again used as explained below (2.6). It can indeed be shown that the operator $(2.15)$ with the elements $(2.19 \mathrm{a})-(2.19 \mathrm{~d})$ is symmetric and positive semidefinite if the operator $D(R)$ is self-adjoint and positive semidefinite 
with respect to the scalar product (2.11). Since the degeneracy requirement (1.15) is satisfied automatically for the choice of transfer coefficients specified in (1.12), the above metric matrix satisfies all requirements of the GENERIC. A comparison of (2.19a)-(2.19d) of the $f$-model with (1.26a)-(1.26d) of the $\boldsymbol{\Phi}$-model highlights the transformation rules under the variable transformation $f \leftrightarrow \boldsymbol{\Phi}$.

The phase change contributions to the dynamic equations of $f(R)$ according to (2.17) thus read

$$
\begin{aligned}
\left.\partial_{t} f(R)\right|_{\mathrm{pc}} & =D(R)\left(\mathbf{a}_{\mu} \cdot \frac{\delta S}{\delta \mathbf{x}^{\mathrm{m}}} \frac{\delta \Phi_{\mu}}{\delta f(R)}+\frac{\delta S}{\delta f(R)}\right) \\
& =D(R)\left(\Lambda_{\mu}^{(S)} \frac{\delta \Phi_{\mu}}{\delta f(R)}-k_{\mathrm{B}}\left(\ln \left(\frac{f(R)}{4 \pi R^{2}}\right)+1\right)\right),
\end{aligned}
$$

whereas the phase change contributions to $\mathrm{x}^{\mathrm{m}}$ are then given through $(2.16 \mathrm{~b})$. For the second equality, the definition (1.21b) was inserted. We notice immediately that, similar to the $\boldsymbol{\Phi}$-model, the functions $\Lambda_{\mu}^{(S)}$ play again the role of driving forces in the $f(R)$-model. The consequences of this similarity shall be examined in more detail below. Furthermore, the term proportional to $k_{\mathrm{B}}$ will result in fluctuations which vanish in the limit $k_{\mathrm{B}} \rightarrow 0$ as shown below.

We now proceed to specify the operator $D(R)$ used in (2.21). According to the declared goal of accounting for fluctuations in the $f(R)$-model (primarily for the nucleation step), and due to the form of the equation for $\left.\partial_{t} f(R)\right|_{\mathrm{pc}}$, the operator $D(R)$ should contain two derivatives with respect to $R$. Furthermore, one can follow the common interpretation that $\left.\partial_{t} f(R)\right|_{\mathrm{pc}}$ is the (negative) divergence of a probability current in configuration space if the leftmost operator in $D(R)$ is $\frac{\partial}{\partial R}$. Due to the positivity of $D(R)$ with respect to the scalar product (2.11) we propose

$$
D(R)=-\frac{\partial}{\partial R} \frac{c_{0}}{2} \frac{f(R)}{4 \pi R^{2}} \frac{\partial}{\partial R},
$$

with the rate constant $c_{0}\left[\mathrm{~m}^{4} \mathrm{~K} \mathrm{~s}^{-1} \mathrm{~J}^{-1}\right]$ with $c_{0} \geq 0$. This rate constant may be an arbitrary function of all variables, $c_{0}\left(\mathbf{x}^{\prime}\right)$; e.g., it may depend on the temperature of the liquid phase to account for the mobility of the single molecules. The factor $f(R)$ ensures linearity of the diffusion equation with respect to the explicit occurrence of $f(R)$. The term $1 / 4 \pi R^{2}$ is due to using the label $R$ instead of $v=\frac{4 \pi}{3} R^{3}$ and represents the fact that attachment and detachment of molecules occurs only at the cluster surface but not inside of the cluster (see the appendix for details). The choice (2.22) for $D(R)$ converts (2.21) into

$$
\left.\partial_{t} f(R)\right|_{\mathrm{pc}}=-\frac{\partial}{\partial R} \frac{c_{0}}{2}\left(\frac{f(R)}{4 \pi R^{2}} \Lambda_{\mu}^{(S)} \frac{\partial}{\partial R} \frac{\delta \Phi_{\mu}}{\delta f(R)}-k_{\mathrm{B}} \frac{\partial}{\partial R} \frac{f(R)}{4 \pi R^{2}}\right) .
$$

Whereas the form (2.21) is sufficient and convenient to study properties of the steady state distribution, the identification of the drift and diffusion term in the diffusion equation can be achieved only through (2.23a). According to stochastic calculus, (2.23a) has a corresponding stochastic differential equation [27, 28], namely

$$
d R=\frac{c_{0}}{2} \Lambda_{\mu}^{(S)}\left(\frac{1}{4 \pi R^{2}} \frac{\partial}{\partial R} \frac{\delta \Phi_{\mu}}{\delta f(R)}\right) d t+\sqrt{\frac{c_{0} k_{\mathrm{B}}}{4 \pi R^{2}}} d W(t),
$$

with $W(t)$ a one-component Wiener process, that is, a Gaussian process with first and second moment given by

$$
\langle W(t)\rangle=0, \quad\left\langle W(t) W\left(t^{\prime}\right)\right\rangle=\min \left(t, t^{\prime}\right) .
$$


Let us now specifically consider the $R$-dependencies in (2.23a) and (2.23b), respectively. If we use $\boldsymbol{\Lambda}^{(S)}$ given by (1.23b) and with

$$
\begin{aligned}
& \frac{\delta \phi}{\delta f(R)}=(1-\phi) \frac{4 \pi}{3} R^{3}, \\
& \frac{\delta \psi}{\delta f(R)}=(1-\phi) 4 \pi R^{2}-\psi \frac{4 \pi}{3} R^{3}
\end{aligned}
$$

for the Boolean grain model, we find

$$
\begin{aligned}
\left.\partial_{t} f(R)\right|_{\mathrm{pc}} & =-\frac{\partial}{\partial R} \frac{c_{0}}{2}\left(\left(A-\frac{B}{R}\right) f(R)-k_{\mathrm{B}} \frac{\partial}{\partial R} \frac{f(R)}{4 \pi R^{2}}\right), \\
d R & =\frac{c_{0}}{2}\left(A-\frac{B}{R}\right) d t+\sqrt{\frac{c_{0} k_{\mathrm{B}}}{4 \pi R^{2}}} d W(t),
\end{aligned}
$$

with

$$
\begin{aligned}
& A=(1-\phi) \Lambda_{0}^{(S)}-\psi \Lambda_{1}^{(S)}, \\
& B=-2(1-\phi) \Lambda_{1}^{(S)} .
\end{aligned}
$$

If the surface tension was absent (i.e., $\Lambda_{1}^{(S)}=0$ ), the drift term in $(2.26 \mathrm{~b})$ would be constant with respect to $R$. As a result the deterministic contribution to the radial growth rate of clusters would be independent of size, as it was primarily suggested in (1.6a)-(1.6c). In the presence of surface tension, however, as in the result for the $\mathbf{\Phi}$ model given in (1.27a), the deterministic radial growth rate becomes size-dependent. Note that these physically intuitive results are based on discussing carefully the explicit $R$-dependences of the entropy and the diffusion operator as done prior and after to (2.4) and (2.22), respectively. We also notice that the diffusive term proportional to $k_{\mathrm{B}}$ in $(2.26 \mathrm{~b})$ becomes less important the larger the crystallite and that fluctuations vanish in the limit $k_{\mathrm{B}} \rightarrow 0$. Furthermore, the first term in the growth equations $(2.26 \mathrm{a}),(2.26 \mathrm{~b})$ illustrates that $c_{0}$ sets the time scale of the process. Whereas the combination of $A(2.27 \mathrm{a})$ and $B(2.27 \mathrm{~b})$ accounts for the thermodynamic driving force and differentiates between crystallization and melting conditions, the rate constant $c_{0}$ may account for kinetic effects such as temperature dependent mobility and structural rearrangement of single molecules prior to their attachment to the growth surface.

The diffusion equation (2.23a) corresponds to the models for nucleation developed by Zeldovich [29] and Frenkel [23], and obtained by Ziabicki [26, 30] as a specific case of a model for more generally shaped clusters. For an introduction, the reader is also referred to [24, 25]. However, in these models the drift term in the diffusion equation is given by the derivative of the free energy gain (with bulk and surface contributions), in contrast to the corresponding, entropy-related term in $(2.23 \mathrm{a}),(2.23 \mathrm{~b})$, which is connected to $\Lambda^{(S)}$. This difference originates from studying close systems here, contrary to open systems in a heat bath in the other models. There are two features of the model developed here without analogy in the other models. The first is the intimate relation between the drift term to the transfer coefficients in the balance equations (1.9a)-(1.9c). And, second, the expressions for $A(2.27 \mathrm{a})$ and $B(2.27 \mathrm{~b})$ indicate that in the very early stages $A \rightarrow \Lambda_{0}^{(S)}$ and $B \rightarrow \Lambda_{1}^{(S)}$, whereas in the late stages $A \rightarrow 0$ and $B \rightarrow 0$ since $\phi \rightarrow 1$ and $\psi \rightarrow 0$. Thus, the cluster growth as described by diffusion equation (2.23a) has an upper limit in the late stages of crystallization. This is remarkable since the distribution function $f(R)$ itself describes objects that may 
overlap arbitrarily; however, it still feels the physical space filling through the dynamics. It should go without saying that the evolution of the system under consideration depends to a high degree on the initial condition and the two boundary conditions imposed on $f(R)[25]$. As the primary interest here is the structure of the equations, we shall not elaborate further on this point.

3. Nucleation and growth rate of supernuclei and crystallites. The diffusion equation (2.26a) describes the time evolution of the distribution $f(R)$ of spherical objects. In this section, the meaning and implications of this equation are studied in more detail. In particular, we are interested in studying the nucleation rate and to what extent the Schneider rate equations (1.6a)-(1.6c) are related to the diffusion equation (2.23a) and (2.26a), respectively.

It has been mentioned above that the diffusion equation (2.23a) is analogous to the one used in nucleation models $[23,29,26,30]$, where the distribution function $f(R)$ describes the number of so-called embryos, critical nuclei, and supernuclei. The use of such terms originates from inspection of the drift term in (2.23a) or (2.26a). Since under favorable conditions for crystallization $A>0$ and $B>0$, there is a critical radius

$$
R^{\star}:=B / A>0,
$$

below which the drift term works to decrease cluster size, whereas above cluster growth is favored. Clusters with $R<R^{\star}$ are then called "embryos" or "subnuclei"; for $R=R^{\star}$ we have "critical nuclei" and for $R>R^{\star}$ they are called "supernuclei." The latter are in the present work referred to as "crystallites," as they can grow spontaneously under the influence of the radial growth rate as discussed in the previous section. Since the Schneider rate equations (1.6a)-(1.6d) are meant for stable clusters (i.e., supercritical nuclei), we aspire in the following to set up dynamic equations for the quantities

$$
\begin{array}{rlr}
\Psi_{\mu}^{\star}(t) & :=\frac{8 \pi}{(3-\mu) !} \int_{R^{\star}(t)}^{\infty} R^{(3-\mu)} f(R, t) d R \\
& \equiv \frac{8 \pi}{(3-\mu) !}\left\langle R^{(3-\mu)}\right\rangle_{f}^{\star} \quad(\mu=0,1,2,3),
\end{array}
$$

in analogy to (1.28), and also for $\phi^{\star}$ and $\psi^{\star}$ given by (1.4a), (1.4b) using $\Psi_{\mu}^{\star}$ instead of $\Psi_{\mu}$. This should allow direct comparison with (1.6a)-(1.6d). For the following it is assumed that $\lim _{R \rightarrow \infty} f(R)=0$, in contrast to the work interested exclusively in the distribution of embryos and the generation of stable nuclei. There the distribution is cut off at a finite upper integration limit due to the limited availability of material (molecules) [25]. Here, however, we shall not make this assumption since our starting point was a distribution function which includes all kinds of clusters, and only as a result of the procedure do we see that not all of them are stable. Using the diffusion equation $(2.26 \mathrm{a})$ we find

$$
\left.\partial_{t} \Psi_{3}^{\star}\right|_{\mathrm{pc}}=-8 \pi\left(\left.f(R, t)\right|_{R=R^{\star}(t)} \partial_{t} R^{\star}(t)+\left.\frac{c_{0} k_{\mathrm{B}}}{2} \partial_{R}\left(\frac{f}{4 \pi R^{2}}\right)\right|_{R=R^{\star}(t)}\right) .
$$

Whereas the first contribution, termed "athermal" (e.g. [26]), is due to changes in the relative thermodynamic state between the phases (i.e., in $A$ and $B$ ) in time, the proportionality to $k_{\mathrm{B}}$ of the second contribution clearly evidences the crucial influence of fluctuations for nucleation. It represents the diffusive current across the 
barrier maximum and is called "thermal nucleation rate" (e.g., [26]). Comparison to (1.6d) identifies the bracket term in (3.3) as the nucleation rate, expressed in terms of a more detailed description than in the $\boldsymbol{\Phi}$-model. Therefore, the result (3.3) and its interpretation clearly must lack any analogy to (1.27b), since in that previous model neither the differently sized clusters are analyzed nor are fluctuations included. For a more detailed analysis of the athermal and thermal nucleation rates and their application, the reader is referred to [30].

Let us now proceed to determine the phase change contributions to the quantities $\Psi_{\mu}^{\star}(\mu=0,1,2)$ defined in $(3.2)$, and $\phi^{\star}$ and $\psi^{\star}$, respectively. Again using the diffusion equation $(2.26 \mathrm{a})$ we find

$$
\begin{aligned}
\left.\partial_{t} \Psi_{\mu}^{\star}\right|_{\mathrm{pc}}= & -\left.\frac{8 \pi}{(3-\mu) !}\left(R^{(3-\mu)} f(R)\right)\right|_{R=R^{\star}(t)} \partial_{t} R^{\star}(t) \\
& +\frac{c_{0}}{2} \frac{8 \pi}{(2-\mu) !}\left(A\left\langle R^{2-\mu}\right\rangle^{\star}-B\left\langle R^{1-\mu}\right\rangle^{\star}\right) \\
& +\frac{k_{\mathrm{B}} c_{0}}{2} \frac{8 \pi}{(3-\mu) !} \int_{R^{\star}(t)}^{\infty} R^{(3-\mu)} \partial_{R}^{2}\left(\frac{f(R)}{4 \pi R^{2}}\right) d R .
\end{aligned}
$$

Similar to the nucleation rate expression (3.3), the first term in (3.4) originates from changes in the thermodynamic states of the phases, whereas the third is again due to the diffusive flux in $R$-space. In the $\boldsymbol{\Phi}$-model developed in [13], first, all clusters down to $R \rightarrow 0$ are included and, second, fluctuations are absent. Considering (3.4), a comparison with the $\boldsymbol{\Phi}$-model must hence be limited to the second term, which is always positive if $A>0$. Using only the latter in (3.4), we find

$$
\begin{aligned}
& \left.\partial_{t} \phi^{\star}\right|_{\mathrm{pc}}=\frac{c_{0}}{2}\left(1-\phi^{\star}\right)\left(\Lambda_{0}^{(S)}+\frac{\mathcal{L}}{\psi} \Lambda_{1}^{(S)}\right) \psi^{\star}, \\
& \left.\partial_{t} \psi^{\star}\right|_{\mathrm{pc}}=\frac{c_{0}}{2}\left(1-\phi^{\star}\right)\left(\Lambda_{0}^{(S)}+\frac{\Psi_{1}^{\star 3}-2 \Psi_{1}^{\star} \Psi_{2}^{\star}+2 \Psi_{3}^{\star}}{\Psi_{2}^{\star}-\Psi_{1}^{\star 2}} \Lambda_{1}^{(S)}\right) \mathcal{L}^{\star}, \\
& \left.\partial_{t} \Psi_{2}^{\star}\right|_{\mathrm{pc}}=\frac{c_{0}}{2}\left(1-\phi^{\star}\right)\left(\Lambda_{0}^{(S)}+\left(\frac{16 \pi\langle 1 / R\rangle^{\star}}{\Psi_{3}^{\star}}-\Psi_{1}^{\star}\right) \Lambda_{1}^{(S)}\right) \Psi_{3}^{\star},
\end{aligned}
$$

where $\mathcal{L}^{\star}$ corresponds to (1.7) using $\Psi_{\mu}^{\star}$ instead of $\Psi_{\mu}$. Equations (3.5a)-(3.5c) have been written in a suggestive form, since the prefactors to $\psi^{\star}, \mathcal{L}^{\star}$, and $\Psi_{3}^{\star}$ in the above equations should all be equal and correspond to the radial growth rate $G$ according to the Schneider rate equations (1.6a)-(1.6c). However, we observe that only the radial growth rate resulting from the $\phi^{\star}$-equation is the same as derived in the $\boldsymbol{\Phi}$-model. In the other two equations, the prefactor collapses only to the same growth rate if the cluster size distribution $f(R)$ is monodisperse, as can be shown by straightforward calculation. Hence, the discrepancy in growth rates in the three equations (1.6a)(1.6c) is specifically due to the larger amount of information contained in the $f$ model. This could certainly not be resolved in the $\boldsymbol{\Phi}$-model, where only the zeroth, first, second, and third moments of the distribution function were considered. It becomes clear from (3.5a)-(3.5c) that the term, which hinders the identification of a single radial growth for all three equations, is again the surface contribution $\Lambda_{1}^{(S)}$. According to (3.4), it is this term which connects the rate of change in the $k$ th moment not only to the $(k-1)$ th but also to the $(k-2)$ th moment. Furthermore, we note that the set of equations are not closed in terms of $\Psi_{\mu}^{\star}$ due to the appearance of $\langle 1 / R\rangle^{\star}$ in (3.5c). 
The above discussion was based on assuming that the formation of the solid phase was favorable, i.e., on $A>0$. If the thermodynamic states of the two competing bulk phases change in such a way that $A \searrow 0$, we have $R^{\star} \rightarrow \infty$ due to (3.1). In other words, all existing structures become unstable and degrade due to the negative overall growth rate originating from the surface tension in $(2.26 \mathrm{a}),(2.26 \mathrm{~b})$. If, in addition, we even have $A<0$, the degradation is enhanced by the bulk contributions. Hence, while the study of the quantities $\Psi_{\mu}^{\star}$ and their dynamics shed light on the nucleation process and the analogy to the $\boldsymbol{\Phi}$-model in the case of growth, the "melting" is more naturally considered on the level of the distribution function $f(R)$.

4. Conclusions and discussion. We have developed a model for liquid-solid/ solid-liquid phase transitions in which the microstructure is described by the Boolean grain model of integral geometry with spherical grains. The model also allows us to control the thermodynamic state of the competing bulk phases and of the interface, whereas the morphology is described by the cluster size distribution function.

A diffusion equation was set up for the cluster size distribution function, and the analogy to the phase change dynamics of coarse-grained variables, namely $\Phi_{\mu}^{\star}$ ( $\mu=0,1,2,3)$, was established. The driving forces for phase change were found to be the same for the $\boldsymbol{\Phi}$ - and the $f$-model. However, the coarse-grained variables have different radial growth rates, which is in clear contrast to the independently formulated earlier $\mathbf{\Phi}$-model [13], in which there is only a single growth rate. It has been discussed above that the difference in coarse-grained growth rates vanishes for a monodisperse distribution, and hence is a polydispersity effect. However, one might wonder why the $\boldsymbol{\Phi}$-model could be tuned towards a single radial growth rate, whereas this could not be enforced by the $f$-model developed here. The reason is the following. It can be shown that in the $\boldsymbol{\Phi}$-model, the entropy production rate per unit volume due to phase change is given by

$$
\left.\sigma\right|_{\mathrm{pc}}=\left.\frac{\delta S}{\delta \mathbf{x}} \cdot \partial_{t} \mathbf{x}\right|_{\mathrm{pc}}=\frac{\delta S}{\delta \mathbf{x}} \cdot \sum_{\mu=0}^{3} \mathbf{a}_{\mu} \Upsilon_{\mu}=\sum_{\mu=0}^{3} \Lambda_{\mu}^{(S)}(\mathbf{x}) \Upsilon_{\mu}(\mathbf{x})
$$

We hence see that $\left\{\Lambda_{\mu}^{(S)}, \Upsilon_{\mu}\right\}$ is a force-flux pair for phase change dynamics, where $\Upsilon_{\mu}$ denotes the phase change in $\Phi_{\mu}$ according to (1.8). It is essential to remember that the four variables $\Phi_{\mu}(\mu=0,1,2,3)$ are independent. Hence, setting up a linear relation between the phase change contribution in $\Phi_{\mu}$ (i.e., the fluxes) and all forces $\Lambda_{\nu}^{(S)}$ as in (1.24) corresponds to the classical procedure with phenomenological coefficients $A_{\mu \nu}$. Whereas the freedom to choose these coefficients was used in the $\boldsymbol{\Phi}$-model to enforce a single radial growth rate, this cannot be transferred to the $f$-model due to the lack of sufficiently many independent variables. However, having developed the consequences of the $f$-model on the dynamics of the coarse-grained variables $\Phi_{\mu}^{\star}$, the choice for $A_{\mu \nu}$ is supported by the more detailed $f$-model.

Appendix. The definition of the entropy contribution due to a distribution function is a subtle problem associated with the proper counting of states. For a distribution function $\tilde{f}(v)$ one is tempted to write down the well-known BoltzmannGibbs entropy expression

$$
S=-k_{\mathrm{B}} \int \tilde{f}(v) \ln \tilde{f}(v) d v .
$$

It can be shown that this entropy used in the GENERIC for formulating a diffusion equation tends to make the function $\tilde{f}(v)$ constant in the absence of drift terms. 
However, if we change variables, $v=v(R)$, the above entropy reads

$$
S=-k_{\mathrm{B}} \int \tilde{f}(v(R)) \frac{d v(R)}{d R} \ln \tilde{f}(v(R)) d R .
$$

Using the definition for the new distribution function

$$
f(R):=\tilde{f}(v(R)) \frac{d v(R)}{d R},
$$

the functional form of the entropy differs from (A.1), namely,

$$
S=-k_{\mathrm{B}} \int f(R) \ln \left(\left.f(R) \frac{d R}{d v}\right|_{v(R)}\right) d R .
$$

In the present study of the cluster size distribution function, we adopt the assumption implicitly made in nucleation theory $[23,25,26]$, namely, that the cluster volume $v=\frac{4 \pi}{3} R^{3}$ and its distribution function $\tilde{f}(v)$ are more fundamental than $R$ and $f(R)$. The reason for doing so is that the very process of phase transition involves attachment/detachment of single molecules, i.e., of volume quanta. The change in radius is then merely a result from changing the volume. However, $R$ is more convenient than $v$ for practical purposes, e.g., to express the radius and the surface of a cluster. Considering then spherical clusters, the entropy for the distribution function for cluster radii $f(R)$ given by (A.4) is then identical to (2.4).

Considering $v$ instead of $R$ as the basic label for the distribution function has ramifications also for the discussion about the diffusion operator $D(R)$ in $(2.21)$, as given prior to $(2.22)$. Thus in the $v$-formulation, $D(v)$ should contain two derivatives $\frac{\partial}{\partial v}$, one of which is the leftmost contribution to $D(v)$ so that the rate of change in $f(v)$ is the divergence of a probability current in configuration space. Due to the positivity of $D(v)$ with respect to the scalar product

$$
\langle A, B\rangle:=\iint_{0}^{\infty} A(\mathbf{r}, v) B(\mathbf{r}, v) d v d^{3} r
$$

and in order to obtain a diffusion equation explicitly linear in $f(v)$, we propose

$$
D(v)=-\frac{\partial}{\partial v} \frac{c_{0}^{\prime}}{2} f(v) v^{2 / 3} \frac{\partial}{\partial v}
$$

with the rate constant $c_{0}^{\prime} \geq 0$. The factor $v^{2 / 3}$, which is proportional to the surface of a cluster of size $v$, is essential. The physics behind this is that clusters can change only their size through surface "reactions"; i.e., the probability current is proportional to the specific cluster surface $v^{2 / 3}$. For more details on the proportionality factor, the reader is referred to, e.g., [23]. Using the chain rule and $v=\frac{4 \pi}{3} R^{3}$, the expression for $D(v)$ given by (A.6) transforms into

$$
D(R)=-\frac{\partial}{\partial R} \frac{c_{0}}{2} \frac{f(R)}{4 \pi R^{2}} \frac{\partial}{\partial R}
$$

with an appropriate constant factor between $c_{0}$ and $c_{0}^{\prime}$.

Acknowledgment. The author thanks Hans Christian Öttinger for valuable discussions and comments. 


\section{REFERENCES}

[1] A. N. Kolmogorov, On the statistical theory of the crystallization of metals, Bull. Acad. Sci. USSR Mat. Ser., 1 (1937), pp. 355-359.

[2] W. A. Johnson And R. F. Mehl, Reaction kinetics in processes of nucleation and growth, Trans. Am. Inst. Min. Engrs., 135 (1939), pp. 416-458.

[3] M. Avrami, Kinetics of phase change. I. General theory, J. Chem. Phys., 7 (1939), pp. 11031112.

[4] M. Avrami, Kinetics of phase change. II. Transformation-time relations for random distribution of nuclei, J. Chem. Phys., 8 (1940), pp. 212-224.

[5] M. Avrami, Kinetics of phase change. III. Granulation, phase change, and microstructure, J. Chem. Phys., 9 (1941), pp. 177-184.

[6] K. R. MEcke, Additivity, convexity, and beyond: Applications of Minkowski functionals in statistical physics, in Statistical Physics and Spatial Statistics: The Art of Analyzing and Modeling Spatial Structures and Pattern Formation, K. R. Mecke and D. Stoyan, eds., Springer, Berlin, 2000, pp. 111-184.

[7] W. Schneider, A. Köppel, And J. Berger, Non-isothermal crystallization of polymers, Int. Polym. Proc., 2 (1988), pp. 151-154.

[8] G. EDER, Mathematical modelling of crystallization process as occurring in polymer processing, Nonlinear Anal., 30 (1997), pp. 3807-3815.

[9] G. Eder and H. Janeschitz-Kriegl, Crystallization, in Materials Science and Technology, Vol. 18, H. Meijer, ed., Wiley-VCH, Weinheim, 1997, pp. 269-342.

[10] M. Ishi, Thermo-Fluid Dynamic Theory of Two-Phase Flow, Eyrolles, Paris, 1975.

[11] D. A. Drew and S. L. Passman, Theory of Multicomponent Fluids, Springer, New York, 1999.

[12] J. Ni AND C. BeCKermann, A volume-averaged two-phase model for transport phenomena during solidification, Metall. Trans. B, 22 (1991), pp. 349-391.

[13] M. HÜTTER, Thermodynamically consistent incorporation of the Schneider rate equations into two-phase models, Phys. Rev. E, 64 (2001), 011209.

[14] M. HÜTTER, GENERIC treatment of compressible two-phase flow: Convection mechanism of scalar morphological variables, J. Non-Equil. Thermodyn., 27 (2002), pp. 349-365.

[15] H. Enwald, E. Peirano, And A.-E. Almstedt, Eulerian two-phase flow theory applied to fluidization, Int. J. Multiphase Flow, 22 (1996), pp. 21-66. (Suppl.)

[16] V. C. Prantil and P. R. Dawson, Application of a mixture theory to continuous casting, in Transport Phenomena in Materials Processing, ASME HTD Vol. 29, M. M. Chen, J. Mazumder, and C. L. Tucker, eds., ASME, New York, 1983, pp. 47-54.

[17] V. C. Prantil and P. R. Dawson, Comparison of a mixture formulation with a similarity solution for binary alloy solidification, Internat. J. Engrg. Sci., 25 (1987), pp. 1283-1304.

[18] D. A. Drew, Mathematical modeling of two-phase flow, Ann. Rev. Fluid Mech., 15 (1983), pp. 261-291.

[19] M. Grmela and H. C. Öttinger, Dynamics and thermodynamics of complex fluids. I. Development of a general formalism, Phys. Rev. E, 56 (1997), pp. 6620-6632.

[20] H. C. Öttinger And M. Grmela, Dynamics and thermodynamics of complex fluids. II. Illustrations of a general formalism, Phys. Rev. E, 56 (1997), pp. 6633-6655.

[21] H. C. ÖTtInger, General projection operator formalism for the dynamics and thermodynamics of complex fluids, Phys. Rev. E, 57 (1998), pp. 1416-1420.

[22] H. C. ÖtTINGER, Derivation of two-generator framework of nonequilibrium thermodynamics for quantum systems, Phys. Rev. E, 62 (2000), pp. 4720-4724.

[23] J. Frenkel, Kinetic Theory of Liquids, Clarendon Press, Oxford, UK, 1946.

[24] P. G. Debenedetti, Metastable Liquids: Concepts and Principles, Princeton University Press, Princeton, NJ, 1996.

[25] D. KAshchiev, Nucleation: Basic Theory with Applications, Butterworth-Heinemann, Oxford, UK, 2000.

[26] A. Ziabicki, Generalized theory of nucleation kinetics. I. General formulations, J. Chem. Phys., 48 (1968), pp. 4368-4374.

[27] C. W. Gardiner, Handbook of Stochastic Methods, Springer, Berlin, 1983.

[28] H. C. Öttinger, Stochastic Processes in Polymeric Fluids. Tools and Examples for Developing Simulation Algorithms, Springer, Berlin, 1996.

[29] J. B. Zeldovich, On the theory of new phase formation: Cavitation, Zhur. Eksper. Teor. Fiz., 12 (1942), pp. 525ff (in Russian); Acta Physicochim. URSS, 18 (1943), pp. 1ff (in English).

[30] A. Ziabicki, Generalized theory of nucleation kinetics. II. Athermal nucleation involving spherical clusters, J. Chem. Phys., 48 (1968), pp. 4374-4380. 